Obituary

\section{Thomas Kreis (1952-98)}

\section{Cell biologist}

Among the 229 lives claimed by the crash of the Swissair flight 111 on 3 September 1998 was that of Thomas Kreis, one of the world's leading cell biologists. At 46, he was at the peak of his life and his careera new father, professor and newly appointed chairman of the department of cell biology at the University of Geneva.

Kreis entered cell biology in the 1970s at a time when the discipline was changing radically. Until then, it had mainly involved the morphological description of cells and tissues. With the advent of molecular biology, a small but influential group initiated analysis of the molecular mechanisms responsible for cellular organization by tackling the problem of sorting: how do the thousands of proteins made by each cell find their correct intracellular and extracellular destinations?

Biochemical assays were devised for studying how proteins synthesized by the protein factories in the cytoplasm are directed to different cellular compartments such as the endoplasmic reticulum, Golgi complex, endosomes, lysosomes and plasma membrane. At the same time, cell biologists started to unravel the functions of the various compartments themselves. But the overall picture was static. It was clear that different compartments had to communicate with each other. But how this is done, and how organelles such as the Golgi complex are built and maintained, was unknown. The biochemical and molecular biological approaches to cell biology were inherently reductionist, and of necessity often destroyed cellular context.

It was here that Kreis came into the picture. He was one of the first to set out to study cellular function in the context of living cells. During his $\mathrm{PhD}$ thesis at the ETH in Zurich, 1978-81, he was already microinjecting fluorescently labelled proteins into live cells and following their fate by fluorescence microscopy. This technique was taken further during his postdoctoral year with Joseph Schlessinger and Benjamin Geiger at the Weizmann Institute in Israel. There he continued his analysis of cytoskeletal elements in the cell, the dynamic network of proteins responsible for cell movement and intracellular transport. This was at a time when the war in Lebanon was at its fiercest and his colleagues wondered, sometimes not happily, at his ability to concentrate on his work ("You are forgetting that I am

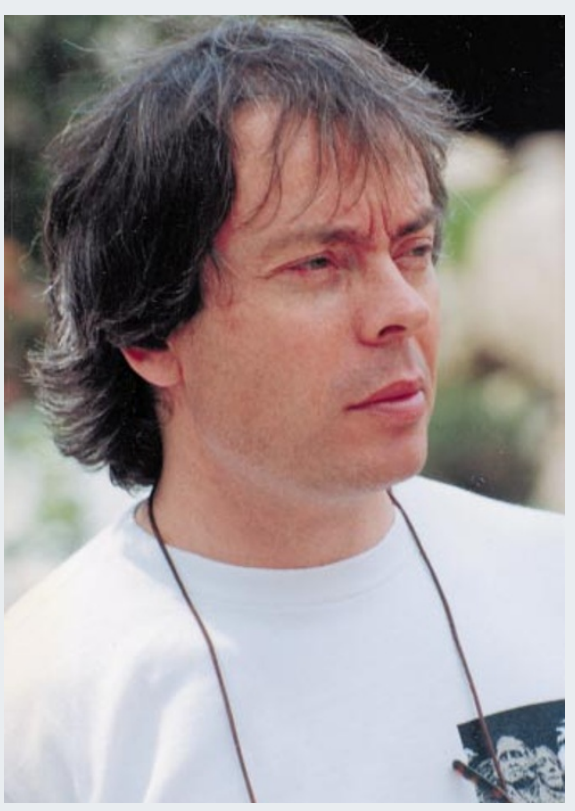

Swiss, and therefore neutral!"). Kreis then spent 1982-83 at the Massachusetts Institute of Technology with Harvey Lodish, a year that influenced him greatly and during which he showed that microinjection could be adapted to probe a wide variety of problems in protein transport.

In 1983, Kreis became a group leader in the cell biology programme at the European Molecular Biology Laboratory, Heidelberg; this was when his science really hit its stride and his reputation became established. Nine years later, at the age of 40 , he moved to Geneva as a professor.

During this 15-year period, Kreis and his group, together with an ever-growing array of international collaborators, established an information base that helped to provide much of our current insight into how intracellular organelles arrange themselves on microtubule tracks and how these tracks are used to transport parcels among compartments in the cell. He was, for instance, the first to establish links between the containers and the microtubules by discovering proteins such as CLIP-170, which connects endosomes to microtubules and so organizes their movements throughout the cell.

In these studies, Kreis might have been inspired by his skill as an expert skier. In one of his last papers, he and his group showed just how intricate the functioning of the cellular transport pathways could be. On the ski slopes, skiers without valid passes sometimes sneak into the gondolas; in the endoplasmic reticulum, proteins not intended for transport to the Golgi complex sometimes sneak into transport vesicles along with proteins actually intended for secretion.

Kreis's imaging of these events in living cells, through the use of reporter molecules labelled with green fluorescent protein, showed that the interlopers are removed from the moving gondolas and packaged into other vesicles that return them to the endoplasmic reticulum. Organizing the entire process is the dynamic network of microtubule cables on which these vesicles move. But what organizes the cables themselves remains a mystery: after depolymerization, microtubules always contrive to reassemble in almost exactly the same pattern.

Kreis's legacy to science extends beyond his discoveries in cell biology to the example set by his style. His approach was characterized by its straightforwardness. As a ski instructor, he used to start his mornings from the top of the highest mountain and then take the straightest route down to the bottom to warm up. Likewise, in research he was always looking for short cuts and quicker ways to the goal; and so, in his dealings with collaborators and colleagues he was honest yet always supportive.

Another element of his style was the understated grace of his work. His beautiful fluorescence micrographs and sensitive hand-drawn images are reflections of a man with a poetic streak (seen also in his extraordinary collection of roses and cactuses).

Kreis also did much for the common good in organizing advanced courses and meetings. Most notably he was one of the initiators of an organization called the European Life Scientist Organization (ELSO). This will start its activities in Geneva in September 2000 with a large congress planned for 3,000-4,000 participants. The hope is that ELSO will become a general forum for catching the excitement in the molecular life sciences and help to raise the standards of biomedical research all over Europe.

Thomas Kreis leaves a wife, son and stepdaughter. He will be missed most by them, but also by the whole community of cell biologists. We have lost, far too prematurely, one of our pioneers.

\section{Kai Simons and Ira Mellman}

Kai Simons is in the Cell Biology Programme, European Molecular Biology Laboratory, Postfach 102209, Meyerhofstrasse 1, 69112 Heidelberg, Germany. e-mail:simons@embl-heidelberg.de Ira Mellman is in the Department of Cell Biology, Yale Medical School, 333 Cedar Street, PO Box 208002, New Haven, Connecticut 06510-8002, USA.

e-mail:ira.mellman@yale.edu 